\title{
Aprendizagem significativa: a teoria como um arcabouço para elaboração de minicursos por acadêmicos de enfermagem*
}

\section{Meaningful learning: theory as a framework for the elaboration of mini-courses by nursing undergraduate students}

\section{Aprendizaje significativo: la teoría como un arcabuz para la elaboración de mini cursos por académicos de enfermería}

Recebido: $19 / 01 / 2018$ Aprovado: 05/10/2018 Publicado: 29/01/2019

\author{
Júnia Lanny Sousa Silva ${ }^{1}$ \\ Larissa Cândida Melo \\ Bibiane Dias Miranda Parreira ${ }^{3}$
}

Este estudo tem como objetivo relatar a experiência de acadêmicos de enfermagem integrantes do Programa de Educação Tutorial, quanto ao planejamento e desenvolvimento de minicursos como atividade extracurricular. Procurou-se descrever como são elaboradas essas atividades numa universidade pública, tendo como arcabouço teórico e metodológico a Teoria da Aprendizagem Significativa, proposta por David Ausubel. Essa teoria destaca duas condições para que a aprendizagem seja significativa, sendo que a primeira se refere à integração de novos conhecimentos aos conhecimentos preexistentes do aprendiz e a segunda à necessidade de que o conteúdo apresentado, seja lógico e psicologicamente significativo. Desta forma, vários são os motivos que levam os estudantes a buscarem atividades extracurriculares como os minicursos, e essa busca exige que os mesmos tenham autonomia e responsabilidade, o que tange também, nesse sentido, a busca por conhecimentos novos e coerentes.

Descritores: Educação em enfermagem; Bases de conhecimento; Aprendizagem.

This study aims at reporting the experience of nursing undergraduate students who are part of the Programa de Educação Tutorial (Program of Tutorial Education), regarding the planning and development of mini-courses as extracurricular activities. It sought to describe how these activities are elaborated in a public university, and had as its theoretical and methodological framework the Theory of Meaningful Learning, proposed by David Ausubel. This theory highlights two conditions for learning to be meaningful. The first is related to the integration between the new knowledge and the previous knowledge of the student, and the second relates to the need for the content being presented to be logical and psychologically meaningful. Thus, there are many reasons that lead students to seek extracurricular activities, such as mini-courses, and this requires them to have autonomy and responsibility, which, therefore, also has a connection to the search for new and coherent knowledge.

Descriptors: Education nursing; Knowledges bases; Learning.

Este estudio tiene como objetivo relatar la experiencia de académicos de enfermería integrantes del Programa de Educación Tutorial, como el planeamiento y desarrollo de mini cursos como actividad extracurricular. Se buscó describir cómo son elaboradas estas actividades en una universidad pública, teniendo como arcabuz teórico y metodológico la Teoría del Aprendizaje Significativo propuesta por David Ausubel. Esta teoría destaca dos condiciones para que el aprendizaje sea significativo, siendo que la primera se refiere a la integración de nuevos conocimientos a los conocimientos preexistentes del aprendiz y la segunda es referente a la necesidad de que el contenido presentado sea lógico y psicológicamente significativo. De esta forma, varios son los motivos que llevan a los estudiantes a buscar actividades extracurriculares como los mini cursos, y esa búsqueda exige que los mismos tengan autonomía y responsabilidad, lo que toca también, en este sentido, la búsqueda por conocimientos nuevos y coherentes.

Descriptores: Educación en Enfermería; Bases del conocimiento; Aprendizaje.

1. Acadêmica do Curso de Enfermagem. Membro do Programa de Educação Tutorial - PET Enfermagem. Universidade Federal do Triângulo Mineiro (UFTM), Uberaba, MG, Brasil. ORCID: 0000-0002-5223-8147 E-mail: juniasssilva@gmail.com

2. Acadêmica do Curso de Enfermagem. Membro do Programa de Educação Tutorial - PET Enfermagem. UFTM, Uberaba, MG, Brasil. ORCID: 0000-0001-6862-300X E-mail: larissa-cmelo@hotmail.com

3. Professora Adjunta do Curso de Enfermagem da UFTM, Uberaba, MG, Brasil. ORCID: 0000-0001-7369-5745 E-mail: bibianedias@yahoo.com.br

* Fonte de Financiamento: Secretaria de Educação Superior do Ministério da Educação/SESU-MEC. 


\section{INTRODUÇÃO}

ransições educacionais, como as do ensino médio para o ensino superior, trazem consigo diversas modificações, que exigem autonomia dos estudantes. Ser autônomo significa que o indivíduo se apresenta claro, seguro e estável de si, com comportamentos estruturados e intencionais na busca por objetivos ${ }^{1}$.

Entende-se que um maior nível de autonomia está associado a uma melhor adaptação acadêmica, referindo-se assim, a quão bem o universitário consegue corresponder às demandas acadêmicas impostas e obter êxito. A autonomia leva o estudante a se conscientizar sobre a importância da busca constante de conhecimentos e da construção de sua identidade social e profissional.

Assim, a presença de autonomia permite também ao discente a busca por conhecimentos, e, consequentemente, por atividades complementares, ou seja, atividades além das que são propostas pela grade curricular convencional, visto que esta muitas vezes apresenta falhas, não oferecendo aos alunos a oportunidade de trabalhar e/ou aprofundar alguns conteúdos e de desenvolver habilidades e conhecimentos importantes na formação profissional. Desse modo, o aluno consegue buscar outros meios de complementar sua graduação, para sanar as falhas encontradas em sua formação, por meio do desenvolvimento de atividades extracurriculares/ complementares ${ }^{2}$.

Os discentes que dedicam mais tempo à dimensão acadêmica, através da participação em atividades extracurriculares, apresentam melhor rendimento acadêmico, menor probabilidade de evasão, mais integração e satisfação no contexto universitário, criação da identidade profissional ${ }^{2}$, além de alavancar a entrada no mercado de trabalho ${ }^{3}$.

As atividades extracurriculares incluem palestras, cursos teóricos, grupos de discussão, disciplinas opcionais, participação em monitorias, congressos, seminários, simpósios, iniciação científica, projetos de ensino, pesquisa e extensão, minicursos, entre outras ${ }^{4}$.

Nesse sentido, os alunos do Programa de Educação Tutorial (PET) de Enfermagem da Universidade Federal do Triângulo Mineiro (UFTM), o PET Enfermagem -, que é desenvolvido por estudantes sob tutoria de docente como forma de atividade complementar/extracurricular e guiado pelos pilares indissociáveis do ensino, pesquisa e extensão -, planejaram e realizaram minicursos, que são eventos de curta duração, visando apresentar uma visão geral sobre um determinado tema vinculado à área de atuação do ouvinte 5 . Os minicursos contribuem positivamente de maneira significativa na formação dos estudantes, buscando levar à ampliação da realização destes no meio acadêmico.

Nesse sentido também, o Programa de Educação Tutorial, conforme preconizado pelo Ministério da Educação, nas Diretrizes Curriculares Nacionais (2001) proporciona atividades que fortaleçam a graduação e contribuam com a formação do discente, em todos os âmbitos ${ }^{5,6}$, além de possibilitar que o conhecimento adquirido pelos estudantes seja disseminado.

Concomitante a isso, Sousa e colaboradores $^{7}$, ressaltam a possibilidade de realização de pesquisas em outras áreas do conhecimento, com vistas a verificar como a educação se faz de forma significativa, permitindo que com esses achados, seja possível a apresentação de novas metodologias, o fortalecimento bem como a divulgação da teoria no campo da saúde.

0 envolvimento com atividades extracurriculares também pode ser suporte para os estudantes universitários durante a graduação, cuja queixa é o distanciamento entre a teoria dada em aula e a prática profissional $^{3}$.

Assim, o presente artigo tem como objetivo relatar a experiência de acadêmicos de enfermagem integrantes do Programa de Educação Tutorial quanto ao planejamento e desenvolvimento de minicursos como atividade extracurricular. 


\section{MÉTODO}

Trata-se de um relato de experiência que tem como referencial teórico a Teoria $d a$ Aprendizagem Significativa, de David Ausubel. A condição do homem de não possuir todo o conhecimento e descobrir que é necessário investir de forma constante na busca e atualização por esse conhecimento e na sua atuação no mundo, constitui-se a base geral do processo educativo ${ }^{7}$.

Utilizou-se a perspectiva qualitativa no formato narrativo-descritivo. As autoras atuaram como protagonistas no desenvolvimento desta experiência, enquanto acadêmicas de enfermagem participantes no Programa de Educação Tutorial de Enfermagem, da Universidade Federal do Triângulo Mineiro - PET Enfermagem. A construção do relato dessa experiência parte da leitura de registros dessas atividades desde sua implantação, em 2006.

Tal fato corrobora a proposta teórica segundo a qual conhecimentos novos devem ser adquiridos a partir de materiais significativos que associem os conhecimentos pré-existentes do aprendiz e o façam construir significados únicos para ele ${ }^{8}$. A aprendizagem significativa consiste em uma abordagem do construtivismo. Os Parâmetros Curriculares Nacionais que datam do ano de 1997, segundo ela, consideram que a aprendizagem, para ser significativa, requer uma associação substantiva entre os conteúdos escolares e os conhecimentos previamente construídos por eles ${ }^{9}$.

Logo, a aprendizagem significativa se caracteriza pela interação entre conhecimentos prévios e novos conhecimentos, sendo que essa interação não deve ser literal e arbitrária, de modo que nesse processo os novos conhecimentos possam adquirir significados para o sujeito e os conhecimentos prévios possam adquirir novos significados.

Para que a aprendizagem seja significativa, duas condições são necessárias: a primeira é referente à disposição do aprendiz para o aprendizado, ou seja, que haja associação entre os conhecimentos novos e os conhecimentos preexistentes de forma não mecânica, evitando a simples memorização dos novos conteúdos, de modo a incorporá-los e associá-los aos conhecimentos que o aprendiz já carrega. Além disto, que queira relacionar os novos conhecimentos a sua estrutura cognitiva prévia, enriquecendo-a e atribuindo significados a esses novos saberes ${ }^{10,11}$.

Como uma segunda condição há necessidade de que o conteúdo apresentado seja lógico, coerente e psicologicamente significativo, permitindo que o aprendiz selecione os conteúdos que ele considera também significativos ou não, a partir das experiências prévias de cada indivíduo. São três as formas de aprendizagem significativa, por subordinação, por superordenação e de modo combinatório ${ }^{10}$.

A aprendizagem subordinada ocorre quando os novos conhecimentos potencialmente significativos adquirem significados para o sujeito que aprende por um processo de apoio, suporte cognitivo em conhecimentos prévios gerais já existentes na sua estrutura cognitiva, ou seja, a pessoa já tem um conhecimento prévio sobre determinado assunto e por meio de um aprendizado significativo essa ideia inicial vai se modificando, se tornando cada vez mais elaborada, sendo capaz de potencializar e servir de ancoradouro cognitivo para novas aprendizagens ${ }^{11}$. 
Figura 1. Aprendizagem significativa: formas e ferramentas.

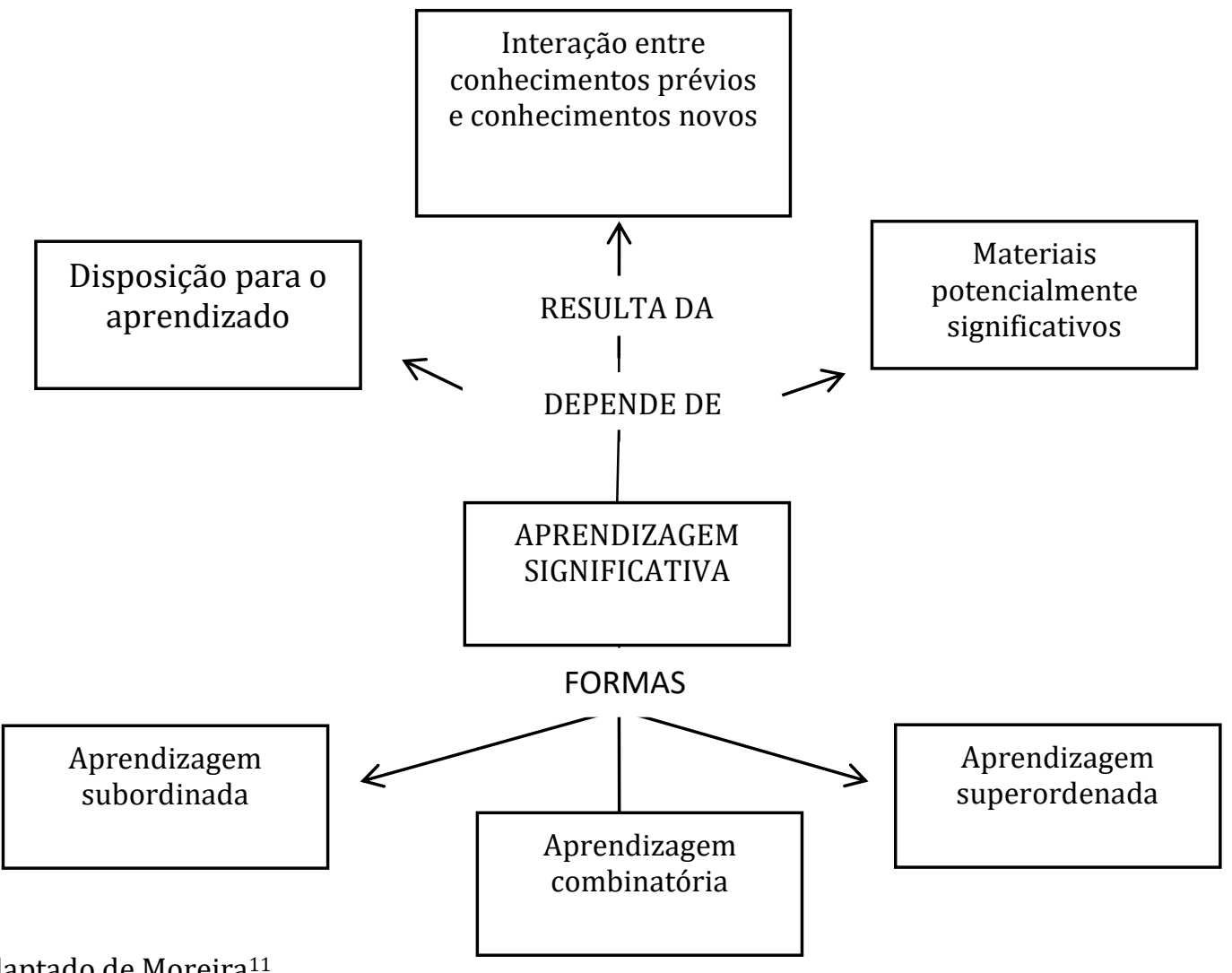

Fonte: Adaptado de Moreira ${ }^{11}$.

A aprendizagem é dita superordenada, quando novos conhecimentos passam a subordinar aqueles que lhe deram origem por um processo de abstração, indução e síntese. Nesse processo, é como se o indivíduo não apresentasse uma ideia geral sobre determinado assunto, e gradualmente por meio de um processo de aprendizagem significativa e de um raciocínio indutivo fizesse associações entre os novos conhecimentos e dessa forma alcançasse o conhecimento ${ }^{11}$.

Já a aprendizagem combinatória, ocorre de forma que a atribuição de significados a um novo conhecimento necessita da interação de diversos outros conhecimentos já existentes na estrutura cognitiva, entretanto, não é mais específica que os conhecimentos originais ${ }^{11}$.

\section{RESULTADOS}

Tendo em vista a preocupação com a transformação, e de uma aprendizagem que integre os conhecimentos prévios do aprendiz aos novos conhecimentos, os alunos do grupo PET Enfermagem organizam os minicursos que compreendem uma atividade realizada desde a fundação na universidade, no ano de 2006.

Fazendo uma retrospectiva dos temas já discutidos e trabalhados, tem-se: periódicos e bases científicas, suporte básico de vida, degustação, PowerPoint, leitura e elaboração de técnicas de texto, terapia infusional, noções básicas de feridas e curativos e oratória.

De cada minicurso organizado, participam em média dezoito a trinta pessoas, incluindo o tutor do grupo, docentes e demais convidados sugeridos.

Desde o primeiro contato com a escolha dos temas a serem ministrados, os discentes integrantes e o tutor do grupo PET Enfermagem sugerem os conteúdos que serão abordados munidos de um mesmo sentimento, que se resume no interesse pelo que será ministrado e no desejo de conectar os novos conteúdos e as experiências e conhecimentos já adquiridos na graduação.

Os temas sugeridos e propostos em grupo são discutidos, de modo que o escolhido seja potencialmente significativo e 
mais do que um tema a ser explicado, desperte a motivação do discente e o faça enxergar a relevância do mesmo para um futuro profissional cuja especialidade está na arte do cuidar.

Os horários para a realização da atividade são escolhidos em consenso com o grupo, na possibilidade da participação de todos, além de ser considerada a disponibilidade dos ministrantes.

Dessa forma, o local da atividade é escolhido mediante o tema acordado. 0 número de encontros e a carga horária para cada minicurso são variados de acordo com a necessidade de tempo do palestrante para abordar cada assunto, mas normalmente variam de um a quatro encontros, de cerca de duas horas cada. Os recursos pedagógicos utilizados também ficam a cargo dos palestrantes, mas, normalmente se usa de data show e/ou debates, de forma dinâmica e interativa.

Ao final de cada atividade todos participantes recebem um certificado, e os integrantes do grupo PET buscam se autoavaliar quanto a seu desempenho, conhecimentos, percepções e expectativas antes e após o realizar de cada minicurso.

Para isso, perguntas norteadoras, elaboradas pelos próprios integrantes do grupo são aplicadas anterior e posterior a atividade. Os "petianos", a partir dos conhecimentos adquiridos, pretendem ser disseminadores destes na sociedade e no próprio ambiente acadêmico, com outros discentes.

\section{DISCUSSÃO}

A aprendizagem ao longo da vida é essencial para os enfermeiros em todos os níveis de prática. Aprender conforme a sua conveniência, frente ao interesse em futuras oportunidades de desenvolvimento profissional, tem sido um tema discutido na atualidade quando se pretende implementar novas estratégias para a educação em enfermagem e o seu desenvolvimento profissional $^{12}$.

Para os discentes de enfermagem, cada vez mais tem sido percebida a necessidade de desenvolvimento e inserção de uma estratégia de ensino-aprendizagem que se afirme de forma a valorizar as experiências relevantes vivenciadas pelo grupo de estudantes, ao longo de sua jornada na formação - haja vista que a busca por conhecimento, se este for compartilhado e discutido com os demais integrantes do grupo, incentiva o interesse e a iniciativa para a integração de conhecimentos, favorecendo $o$ processo ensinoaprendizagem ${ }^{13}$.

As iniciativas para formação, como a relatada, que se utiliza de métodos como a socialização, têm sido consideradas atividades importantes para os discentes, uma vez que lhes permitem compartilhar conhecimentos e experiências de outros, contribuindo para o crescimento pessoal e profissional, sendo erros ou acertos meios para a aprendizagem ${ }^{13}$.

A formação acadêmica tem sido permeada pela integração dos estudantes em outras iniciativas de aprendizagem para além da grade curricular tradicional, como: a realização de palestras, cursos teóricos e grupos de discussão, como a inserção de disciplina opcional ou obrigatória no currículo, bem como o incentivo à pesquisa científica, que favorecem a abordagem, nos mais diferentes aspectos, e a ampliação de conteúdos na formação $0^{4}$.

Deve-se considerar que a enfermagem é uma ciência centrada na pessoa e uma de suas principais competências é ter o forte compromisso com a sociedade, para dar respostas ajustadas às suas necessidades, promovendo a livre escolha dos indivíduos nas decisões de saúde e ajustando-se a princípios bioéticos. Por conseguinte, afirmase que o profissional enfermeiro deve ter em sua formação a aproximação com amplos conhecimentos, de forma a pautarem a qualificação de seu agir ${ }^{14}$.

Considera-se a aprendizagem significativa, nesta abordagem de estratégias para a formação complementar dos futuros enfermeiros, como a capacidade de contribuir para influenciar as mudanças de atitudes nas relações de produção do conhecimento, e propiciar uma aprendizagem impulsionada por mecanismos 
de comunicação que busquem a autonomia nas relações.

$\mathrm{Na}$ atualidade, fatores limitantes ao desenvolvimento e formação profissional apresentam-se pelos modelos de formação propostos, nos quais os enfermeiros não se identificam com a abordagem ensinoaprendizagem, gerando desistên cias ${ }^{15}$.

\section{CONCLUSÃO}

Este trabalho reforça a importância da realização proativa de atividades extracurriculares, como os minicursos. São vários os motivos que levam os estudantes a buscarem essas atividades complementares, busca essa que exige autonomia.

Nesta perspectiva, a atividade extracurricular relacionada aos minicursos, permitiu disponibilizar ao ambiente acadêmico, na formação de enfermeiros, uma iniciativa complementar orientada por um processo de aprendizagem que buscou ampliar significados aos acadêmicos.

Dentre os principais aspectos que favoreceram a aprendizagem significativa nesta experiência tem-se a sugestão coletiva de temas a serem trabalhados, fato que acaba por motivar o discente a se integrar nas atividades. Um estudante autônomo é mais seguro de si e da busca por comportamentos na direção de conhecimentos novos e coerentes.

\section{REFERÊNCIAS}

1. Alves AF, Gonçalves P, Almeida L. Acesso e sucesso no ensino superior: inventariando as expectativas dos estudantes. Rev Galego-Port Psicol Educ. [Internet]. 2012 [citado em 15 jun 2017]; 20(1):121-31. Disponível em: https://ruc.udc.es/dspace/bitstream/handle /2183/12112/RGP_20_2012_art_8.pdf?seque nce $=1 \&$ isAllowed $=y$

2. Fior CA, Mercuri E. Formação universitária e flexibilidade curricular: importância das atividades obrigatórias e não obrigatórias. Psicol Educ. [Internet]. 2009 [citado em 12 ago 2017]; 29(1):191-215. Disponível em: http://pepsic.bvsalud.org/pdf/psie/n29/n29 a10.pdf

3. Oliveira CT, Santos AS, Dias ACG. Percepções de estudantes universitários sobre a realização de atividades extracurriculares na graduação. Psicol Ciênc Prof. [Internet] 2016 [citado em 20 ago 2017]; 36(4):864-76. DOI: http://dx.doi.org/10.1590/1982-

3703003052015

4. Silva NCM, Lunes DH, Resck ZMR, Soares MI, Souza Junior DI, Vieira NF. Estratégias de ensino das terapias alternativas e complementares na graduação em enfermagem: revisão integrativa. Rev Eletrônica Enferm. [Internet] 2013 [citado em 16 jun 2017]; 15(4):1061-7.

DOI:

http://dx.doi.org/10.5216/ree.v15i4.20568

5. Ministério da Educação (Br). Programa de orientação tutorial-PET: manual de orientações básicas. Brasília, DF: Ministério da Educação; 2006. 25p. Disponível em: http://portal.mec.gov.br/index.php?option=c om_docman\&view=download \&alias=338manualorientabasicas\&category_slug=petprograma-de-educacaotutorial\&Itemid $=30192$

6. Fernandes JD, Rebouças LC. Uma década de Diretrizes Curriculares Nacionais para a Graduação em Enfermagem: avanços e desafios. Rev Bras Enferm. [Internet]. 2013 [citado em 15 de mar 2017]; 66(1):95-101. Disponível em:

http://www.scielo.br/pdf/reben/v66nspe/v 66nspea13.pdf

7. Sousa ATO, Formiga NS, Oliveira SHS, Costa MML, Soares MJGO. A utilização da teoria da aprendizagem significativa no ensino da enfermagem. Rev Bras Enferm. [Internet]. 2015 [citado em 16 set 2017]; 68(4):713-22. DOI: http://dx.doi.org/10.1590/0034-

7167.2015680420i

8. Ausubel D. Aquisição e retenção de conhecimentos: uma perspectiva cognitiva. Lisboa: Plátano Edições Técnicas; 2000. Apresentação da teoria da assimilação da aprendizagem e da retenção significativas; v. 1, p.1-19. Disponível em: http://www.uel.br/pos/ecb/pages/arquivos /Ausubel_2000_Aquisicao\%20e\%20retencao $\% 20 \mathrm{de} \% 20$ conhecimentos.pdf

9. Ministério da Educação (Br). Parâmetros Curriculares Nacionais: introdução aos parâmetros curriculares nacionais. Brasília, DF: Ministério da Educação; 1997. Disponível em: 
http://portal.mec.gov.br/seb/arquivos/pdf/l ivro01.pdf

10. Pelizzari A, Kriegl ML, Baron MP, Finck NTL, Dorocinski SI. Teoria da aprendizagem significativa segundo Ausubel. Rev PEC. [Internet]. 2002 [citado em 20 set 2017]; 2(1):37-42. Disponível em: http://portaldoprofessor.mec.gov.br/storage /materiais/0000012381.pdf

11. Moreira MA. Afinal o que é aprendizagem significativa? Rev Qurriculum [Internet]. 2012 [citado em 10 jun 2017]; 25(1):29-56. Disponível em: http://publica.webs.ull.es/upload/REV\%20Q URRICULUM/25\%20-\%202012/02.pdf

12. Nedder MM, Levine SA, Galligan C, Avery KR, Eagan-Bengston E, Reilly KM. Blogging as innovative method of peer-to-peer educational sharing. Crit Care Nurse [Internet]. 2017 [citado em 16 set 2017]; 37(1):1-9. Disponível em: http://ccn.aacnjournals.org/content/37/1/e 1.full.pdf+html. DOI: $10.4037 / \mathrm{ccn} 2017642$.

13. Hermida PMV, Barbosa SS, Heidemann ITSB. Metodologia ativa de ensino na formação do enfermeiro: inovação na Atenção Básica. Rev Enferm UFMS. [Internet]. 2015 [citado em 25 out 2017]; 5(4):683-691. Dispo- nível

em:

https://periodicos.ufsm.br/reufsm/article/vi ew $/ 16920 / p d f$.

DOI:

10.5902/2179769216920

14. Fernández-Cervilla $\mathrm{AB}$, Dorado-Piris $\mathrm{AI}$, Cabrer-Vives ME, Barquero-González A. Estado atual do ensino de terapias complementares na formação superior de enfermagem na Espanha. Rev Latinoam Enferm. [Internet]. 2013 [citado em 25 out 2017]; 21(3):1-8. Disponível em: http://www.scielo.br/pdf/rlae/v21n3/pt_01 04-1169-rlae-21-03-0679.pdf

15. Myra Viljoen RN, Isabel Coetzee RN, Tanya Heyns RN. Critical care nurses' Reasons for poor attendance at a continuous professional development program. Am J Crit Care [Internet]. 2017 [citado em 10 out 2017]; 26(1):70-6. Disponível em: http://ajcc.aacnjournals.org/content/26/1/7 0.full.pdf+html. DOI: $10.4037 /$ ajcc2017412

CONTRIBUIÇÕES
Júnia Lanny Sousa Silva e Larissa Cândida
Melo atuaram na concepção, delineamento,
análise e interpretação dos dados e redação
do artigo. Bibiane Dias Miranda Parreira
atuou na redação do artigo e revisão crítica.

\section{Como citar este artigo (Vancouver)}

Silva JLS, Melo LC, Parreira BDM. Aprendizagem significativa: a teoria como um arcabouço para elaboração de minicursos por acadêmicos de enfermagem. REFACS [Internet]. 2019 [citado em inserir dia, mês e ano de acesso]; 7(1):82-88. Disponível em: inserir link de acesso. DOI: inserir link do DOI.

\section{Como citar este artigo (ABNT)}

SILVA, J. L. S.; MELO, L. C.; PARREIRA, B. D. M. Aprendizagem significativa: a teoria como um arcabouço para elaboração de minicursos por acadêmicos de enfermagem. REFACS, Uberaba, MG, v. 7, n. 1, p. 82-88, 2019. Disponível em: <inserir link de acesso >. Acesso em: inserir dia, mês e ano de acesso. DOI: inserir link do DOI.

\section{Como citar este artigo (APA)}

Silva, J.L.S.; Melo, L.C. \& Parreira, B.D.M. (2019). Aprendizagem significativa: a teoria como um arcabouço para elaboração de minicursos por acadêmicos de enfermagem.

REFACS, 7(1), 82-88. Recuperado em: inserir dia, mês e ano de acesso de inserir link de acesso. DOI: inserir link do DOI. 